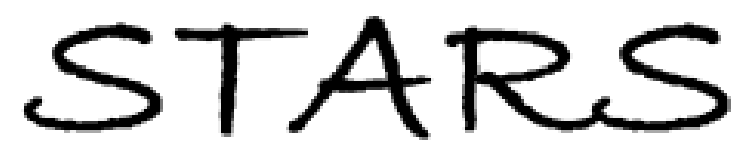

University of Central Florida

STARS

$1-1-2003$

\title{
The role of geometric constraints in amphiphilic self-assembly: A Brownian dynamics study
}

\author{
Geuorgui K. Bourov \\ University of Central Florida \\ Aniket Bhattacharya \\ University of Central Florida
}

Find similar works at: https://stars.library.ucf.edu/facultybib2000

University of Central Florida Libraries http://library.ucf.edu

This Article is brought to you for free and open access by the Faculty Bibliography at STARS. It has been accepted for inclusion in Faculty Bibliography 2000 s by an authorized administrator of STARS. For more information, please contactSTARS@ucf.edu.

\section{Recommended Citation}

Bourov, Geuorgui K. and Bhattacharya, Aniket, "The role of geometric constraints in amphiphilic selfassembly: A Brownian dynamics study" (2003). Faculty Bibliography 2000s. 3634.

https://stars.library.ucf.edu/facultybib2000/3634

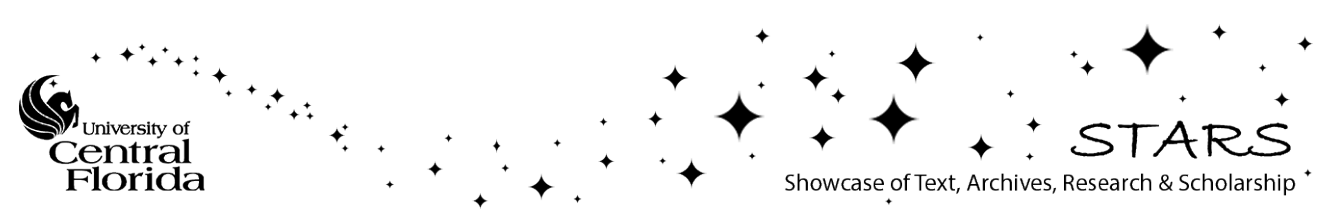




\section{The role of geometric constraints in amphiphilic self-assembly: A Brownian dynamics study}

Cite as: J. Chem. Phys. 119, 9219 (2003); https://doi.org/10.1063/1.1614210

Submitted: 19 May 2003. Accepted: 07 August 2003. Published Online: 17 October 2003

Geuorgui K. Bourov, and Aniket Bhattacharya

\section{ARTICLES YOU MAY BE INTERESTED IN}

Spontaneous curvature of bilayer membranes from molecular simulations: Asymmetric lipid densities and asymmetric adsorption

The Journal of Chemical Physics 142, 054101 (2015); https://doi.org/10.1063/1.4906149

Simulation studies of self-assembly of end-tethered nanorods in solution and role of rod aspect ratio and tether length

The Journal of Chemical Physics 125, 184903 (2006); https://doi.org/10.1063/1.2363983

Brownian dynamics simulation study of self-assembly of amphiphiles with large hydrophilic heads

The Journal of Chemical Physics 122, 044702 (2005); https://doi.org/10.1063/1.1834495

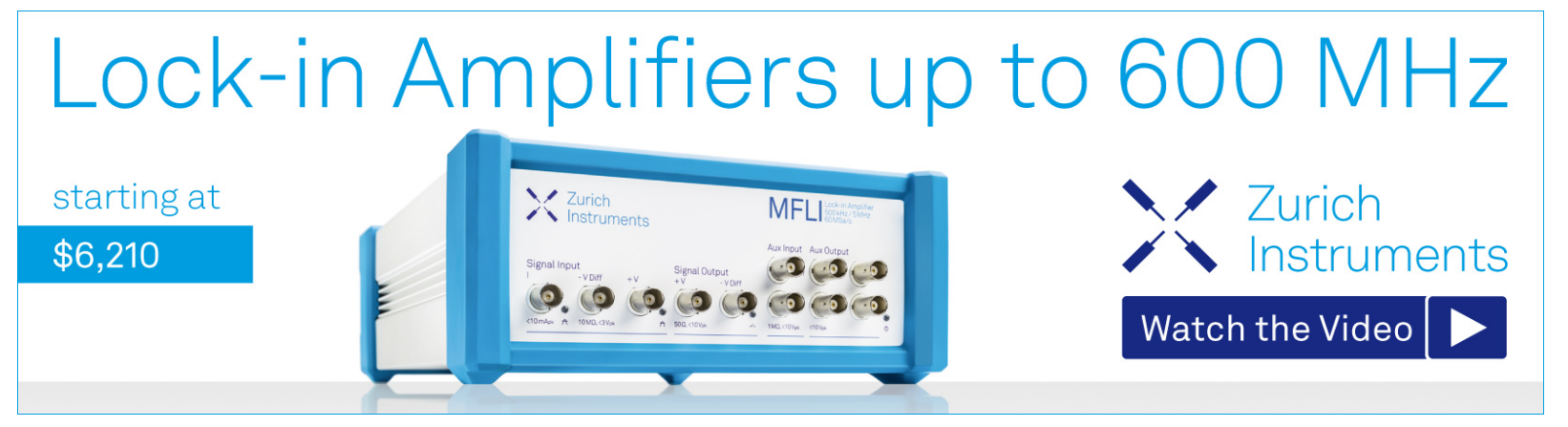

J. Chem. Phys. 119, 9219 (2003); https://doi.org/10.1063/1.1614210 


\title{
The role of geometric constraints in amphiphilic self-assembly: A Brownian dynamics study
}

\author{
Geuorgui K. Bourov and Aniket Bhattacharya ${ }^{\text {a) }}$ \\ Department of Physics, University of Central Florida, Orlando, Florida 32816-2385
}

(Received 19 May 2003; accepted 7 August 2003)

\begin{abstract}
We use a stochastic molecular dynamics simulation method to investigate the effect of optimal head group area in amphiphilic self-assembly. For a fixed tail geometry, we choose several sizes of the effective head group area and carry out a detailed study of how this affects the critical micelle concentration (CMC), the cluster distribution, and the shape of micelles for different concentrations and temperature. We find that with an increase of the effective head group area, the CMC is attained at a larger concentration of the free chains at all temperatures. Likewise, for a given concentration, amphiphiles with the larger effective head group exhibit a sharper cluster distribution with a tendency to form more spherical micelles. Our study shows a way to control the size and shape of the micelles and can have potential impact on the synthesis of nano-structures through surfactant mediated templating methods. (C) 2003 American Institute of Physics. [DOI: 10.1063/1.1614210]
\end{abstract}

\section{INTRODUCTION}

There is an increasing need to understand the selfassembling properties of short amphiphilic chain molecules $^{1,2}$ as they find ample uses in forming templates in the nano-fabrication of various devices. For example, semiconductor nano-structures are synthesized by the use of diblock copolymers as nano-lithographic masks. ${ }^{3}$ Broadly speaking, this is an emergent area where it is believed that the self-assembling properties of amphiphiles and block copolymers can be utilized in the parallel production of devices in nano-meso scales which are otherwise difficult to produce using conventional lithographic techniques. Amphiphilic self-assembly is also relevant for cell biology. ${ }^{4}$ Cell membranes are composed of lipid bilayers which are made off amphiphilic molecules with two hydrophobic tails. The passage time of an individual polynucleotide molecule, e.g., DNA, through an ion channel in lipid bilayer membrane can be used for high speed detection of sequence of bases; ${ }^{5}$ the modes translocation of RNA or DNA across a lipid bilayer is an important and yet unsolved problem in biophysics. The amphiphilic self-assembly has found applications in medicine as well; the pockets formed by magnetic colloids coated with phospolipid vesicles have been identified as drug delivery agents. ${ }^{6}$ It is therefore necessary to understand selfassembly in these soft matter systems at a fundamental level.

The property that makes amphiphilic aggregation unique is its tendency, when dissolved in water, to form a variety of structures; spherical and cylindrical micelles, bilayers, vesicles, disordered and ordered bi-continuous structures are formed depending on the concentration of the amphiphiles, salinity of the solution, and temperature. It has been indicated by Israelachvili ${ }^{1,7}$ that the intrinsic geometry of an individual amphiphile has a strong influence on the final shape of the aggregate or micelle. In three dimensions (3D), it straight forward to show that the various shapes of miceller

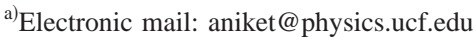

aggregates can be characterized by the dimensionless packing parameter $\lambda_{3 \mathrm{D}}=v / a_{0} l_{c}$, where $v, a_{0}$, and $l_{c}$ are the volume, the optimal head group area, and the critical chain length of an amphiphile respectively. Spherical and nonspherical micelles, and bi-layers are formed for $v / a_{0} l_{c}<\frac{1}{3}, \frac{1}{3}$ $<v / a_{0} l_{c}<\frac{1}{2}$, and $\frac{1}{2}<v / a_{0} l_{c}<1$, respectively. ${ }^{1}$

Simulation studies of various kinds have been carried out in the past to study amphiphilic aggregation and have contributed enormously to our understanding of selfassembly. Both lattice ${ }^{8-17}$ and off-lattice ${ }^{18-21}$ models with and without the explicit incorporation of the solvent particles have been studied; more recently Brownian dynamics simulation has been proven to be quite efficient to study amphiphilic self-assembly. ${ }^{22}$ Despite considerable activities in simulation and modeling of amphiphilic self-assembly, that the geometry is a key factor in self-assembly has not been undertaken in a systematic fashion, although isolated amphiphiles of rather complex geometry have been studied. ${ }^{23}$ In this paper we make a detailed study of how the architecture of a single amphiphile influences its CMC, sizes, and shapes of the micelles. For a fixed length of the hydrophobic tail, we vary the packing parameter by changing the size of the hydrophilic head to increase the effective head group area $a_{0}$ and demonstrate how the geometry of the amphiphile can be used to tailor micelles of specific shapes and sizes. We believe that these studies will be very useful in surfactant mediated methods applied to nanotechnology. The organization for the rest of the paper is as follows. In the next section we describe our model and method. In Sec. III we describe in detail our results. In Sec. IV we provide a summary and discussion of our main results.

\section{MODEL AND NUMERICAL PROCEDURE}

In this paper we have used a stochastic MD simulation method $^{26,27}$ and consider a 2D continuum model in which each amphiphile is represented by a chain consisting of $m$ monomers connected by anharmonic spring potential as de- 
TABLE I. Interaction parameters for the amphiphiles.

\begin{tabular}{lccc}
\hline \hline Interaction & $\frac{r_{i j}^{c}}{\sigma_{i j}}$ & $\sigma_{i j}$ & $\epsilon_{i j}$ \\
\hline Head-head & $2^{1 / 6}$ & $\sigma_{\mathrm{tt}}, 2 \sigma_{\mathrm{tt}}, 3 \sigma_{\mathrm{tt}}$ & 1.0 \\
Head-tail & $2^{1 / 6}$ & $\frac{\sigma_{\mathrm{hh}}+\sigma_{\mathrm{tt}}}{2}$ & 1.0 \\
Tail-tail & 2.5 & 1.0 & 1.0 \\
\hline \hline
\end{tabular}

scribed below. For an amphiphile of length $m$, the first monomer is considered to be the hydrophilic head $(h)$ and the remaining $m-1$ monomers represent the hydrophobic tail particles $(t)$. In general, we will denote an amphiphile with $x$ and $y$ number of head and tail segments as $h_{x} t_{y}$.

The potentials that act on the particles consists of two parts: $U_{\mathrm{LJ}}^{i j}$, and $U_{\text {chain }}$. Here $U_{\mathrm{LJ}}^{i j}$ is a Lennard-Jones (LJ) potential acting between any two pair of monomers $i$ and $j$ :

$$
\begin{aligned}
U_{\mathrm{LJ}}^{i j}\left(r_{i j}\right)= & 4 \epsilon_{i j}\left[\left(\frac{\sigma_{i j}}{r_{i j}}\right)^{12}-\left(\frac{\sigma_{i j}}{r_{i j}}\right)^{6}-\left(\frac{\sigma_{i j}}{r_{i j}^{c}}\right)^{12}+\left(\frac{\sigma_{i j}}{r_{i j}^{c}}\right)^{6}\right] ; \\
r & \leqslant r_{i j}^{c},
\end{aligned}
$$

where $r_{i j}^{c}$ is the cutoff distance beyond which the LJ interaction is set to be zero, $r_{i j}=\left|\vec{r}_{i}-\vec{r}_{j}\right|$ and $\vec{r}_{i}, \vec{r}_{j}$ are the locations of the $i$ th and $j$ th monomers, respectively. Amphiphilicity in this model is introduced by a repulsive cut-off distance for the head-head and head-tail $\left(r_{\mathrm{hh}}^{c}=2^{1 / 6} \sigma_{\mathrm{hh}}, r_{\mathrm{ht}}^{c}=2^{1 / 6} \sigma_{\mathrm{ht}}\right)$, and an attractive cut-off for the tail-tail interaction $\left(r_{\mathrm{tt}}^{c}\right.$ $\left.=2.5 \sigma_{\mathrm{tt}}\right)$. The parameter $\epsilon_{i j}$ is kept to unity for any pair of species. The choice of the LJ parameters are summarized in Table I. $U_{\text {chain }}$ is the Finite-Extendable Nonlinear Elastic (FENE) anharmonic spring potential acting between pairs of successive monomers along a chain:

$$
U_{\text {chain }}\left(r_{i j}\right)=-0.5 k R_{i j} \ln \left[1-\left(\frac{r_{i j}}{R_{i j}}\right)^{2}\right],
$$

in which $k$ and $R_{i j}$ are the energy and the length parameter of the potential.

The MD method that we have implemented here is the same as the one previously employed by one of us ${ }^{24,25}$ and very similar to the method adopted by Grest and co-workers earlier. ${ }^{26}$ To simulate a constant temperature ensemble, the monomers are coupled to a heat bath and the equations of motion read as

$$
\ddot{\vec{r}}_{i}=-\vec{\nabla} U_{i}-\Gamma \dot{\vec{r}}_{i}+\vec{W}_{i}(t),
$$

where

$$
U_{i}=\sum_{i \neq j}\left[\left(U_{\mathrm{LJ}}^{i j}\left(r_{i j}\right)+U_{\text {chain }}\left(r_{i j}\right)\right],\right.
$$

$\Gamma$ is the monomer friction coefficient and $\vec{W}_{i}(t)$ which describes the random force of the heat bath acting on each monomer is a Gaussian white noise with zero mean satisfying the fluctuation-dissipation relation:

$$
\left\langle\vec{W}_{i}(t) \cdot \vec{W}_{j}\left(t^{\prime}\right)\right\rangle=6 k_{\mathrm{B}} T \Gamma \delta_{i j} \delta\left(t-t^{\prime}\right) .
$$

We use the reduced units throughout this study; the unit of time is $\sigma\left(m / \epsilon_{\mathrm{tt}}\right)^{1 / 2}$ and the unit of temperature is $\epsilon_{\mathrm{tt}} / k_{\mathrm{B}}$, where $k_{\mathrm{B}}$ is the Boltzmann constant. The details of the numerical expediency in choosing a fast Gaussian random number generator and a link-cell for force and energy calculation can be found in Refs. 24, 25. We have chosen $k=30$ and $R_{i j}=1.5 \sigma_{i j}$ which make chain crossing practically impossible. ${ }^{26}$

\section{RESULTS}

We present simulation results for amphiphiles of type $h_{1} t_{4}$ and $h_{1} t_{6}$, respectively. Most of the simulations are carried out in a square box of length $100 \sigma_{\mathrm{tt}}$. In order to study the finite size effects we have carried out simulation in a box of length $200 \sigma_{\mathrm{tt}}$. The optimal head group area is varied by choosing different $\sigma_{\mathrm{hh}}$-parameter for the LJ interaction (see Table I) to be $\sigma_{\mathrm{tt}}, 2 \sigma_{\mathrm{tt}}$, and $3 \sigma_{\mathrm{tt}}$ for a fixed length of the hydrophobic tail, or by choosing different length of the hydrophobic tail for a fixed $\sigma_{\mathrm{hh}}$. Depending upon the temperature and concentration, the length of the run varied from $(2-10) \times 10^{6}$ MD steps. The maximum number of chains were 1200 . The simulations were run primarily on a 10 processor Linux cluster.

\section{A. Critical micelle concentration (CMC)}

A characteristic feature of amphiphilic self-assembly is the existence of a CMC beyond which the concentration of free single chains $X_{1}$ ceases to increase. The basic thermodynamic reasonings which are valid at low concentration predicts that at and beyond the CMC $X_{1}$ remains roughly constant as it becomes free energetically favorable to form larger clusters. Various identifying features have been proposed for an accurate characterization of the CMC. ${ }^{13,14,28-31}$ One of us $(\mathrm{AB})$ proposed the onset of a peak in the specific heat to characterize the $\mathrm{CMC},{ }^{13,14}$ which has been used later by others. ${ }^{15}$ Here we study the effect of the architecture of the amphiphile on CMC. Equilibria among different aggregates imply the itsame chemical potential $\mu$ for all the species, which leads to the following equation: ${ }^{1}$

$$
\mu=\mu_{m}^{0}+\frac{k_{\mathrm{B}} T}{m} \log \left(\frac{X_{m}}{m}\right), \quad m=1,2,3, \ldots, \infty,
$$

where $X_{m}$ and $\mu_{m}$ are the concentration of the amphiphiles and the chemical potential of the clusters of size $m$, and $\mu_{m}^{0}$ is the standard part of the chemical potential. It then follows from the above equation that the concentration of the $N$ th species $X_{N} / N$ is expressed as

$$
\frac{X_{N}}{N}=\left[X_{1} \exp \left(\frac{\mu_{1}^{0}-\mu_{N}^{0}}{k_{\mathrm{B}} T}\right)\right]^{N},
$$

where the concentration of chains in each species $X_{i}$ has to satisfy the sum rule

$$
\sum_{i=1}^{\infty} X_{i}=X
$$

Evidently, a change in $X_{1}$ will affect the cluster distribution. A different size of the head geometry will shield the hydrophobic tail segments in a different manner which will also 


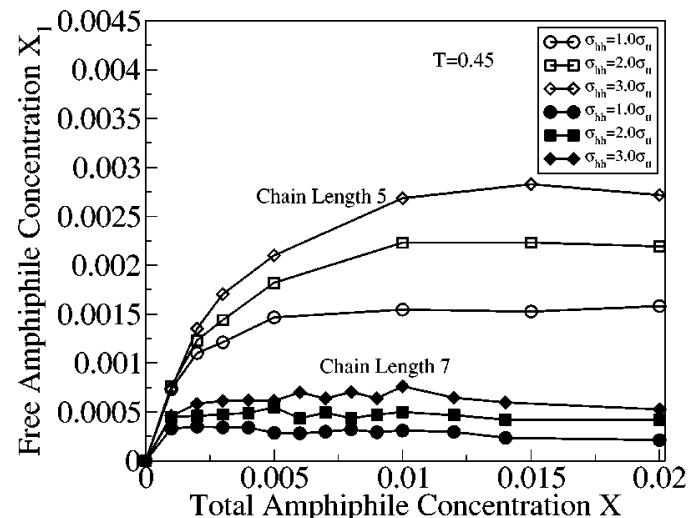

FIG. 1. Variation of free chain concentration $X_{1}$ as a function of the total concentration of amphiphiles $X$ at $T=0.45$ for different head sizes. The open and the closed $\bigcirc, \square$, and the $\diamond$ correspond to $\sigma_{\mathrm{hh}}=\sigma_{\mathrm{tt}}, \sigma_{\mathrm{hh}}=2 \sigma_{\mathrm{tt}}$, $\sigma_{\mathrm{hh}}=3 \sigma_{\mathrm{tt}}$ for $h_{1} t_{4}$ and $h_{1} t_{6}$, respectively.

affect the chemical potential of the clusters. Indeed we notice that the geometry of the head group has a marked effect on the CMC, the shapes, and the distribution of micelles. In Fig. 1 we show the dependence of CMC on amphiphilic geometry by plotting $X_{1}$ as a function of $X$ for three different effective sizes of the hydrophilic head $\sigma_{\mathrm{hh}}$ for two different chain lengths. It is seen from the figure that for a given concentration the amphiphiles with bigger heads are less inclined to form micelles as the $X_{1}$ saturates at a higher value; i.e., the $C M C$ increases with increasing head size, a result that has not been reported earlier.

Figure 2 shows the dependence of CMC for $h_{1} t_{6}$ for two different temperatures. It is observed from this figure that the $\mathrm{CMC}$ for the chain with $\sigma_{\mathrm{hh}}=3 \sigma_{\mathrm{tt}}$ at $T=0.45$ (filled diamonds) effectively moves to the CMC of a chain with the same hydrophobic tail length; but with $\sigma_{\mathrm{hh}}=\sigma_{\mathrm{tt}}$ at $T=0.5$ (open circles). Therefore, to a first approximation, the CMC for a choice of a larger head group is mapped onto the CMC of a smaller head group at a higher temperature. We will see later that although the $X_{1} \sim X$ curves fall one on top of each other, the cluster distributions and shapes of these two systems are very different.

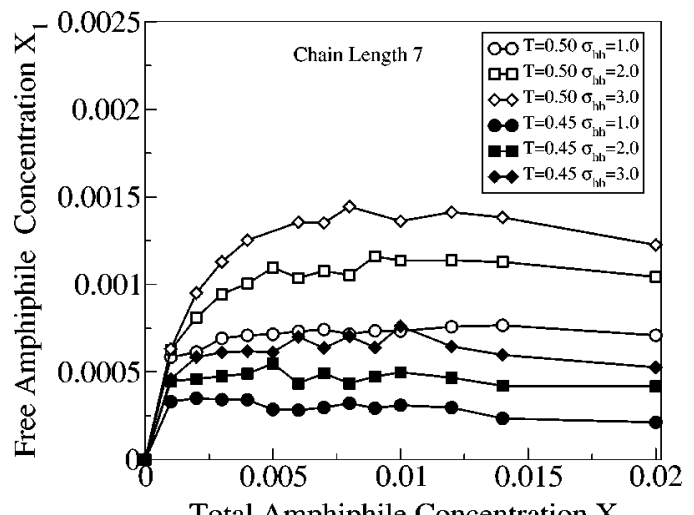

Total Amphiphile Concentration X

FIG. 2. Variation of free chain concentration $X_{1}$ as a function of the total concentration $X$ for $h_{1} t_{6}$. The open and the closed $\bigcirc, \square$, and the $\diamond$ correspond to $\sigma_{\mathrm{hh}}=\sigma_{\mathrm{tt}}, \sigma_{\mathrm{hh}}=2 \sigma_{\mathrm{tt}}, \sigma_{\mathrm{hh}}=3 \sigma_{\mathrm{tt}}$ for $T=0.5$ and $T=0.45$, respectively.
We note from Fig. 2 that beyond the CMC, the value of $X_{1}$ is strictly not flat, but it decreases as the total concentration $X$ is increased. This has been noticed earlier by Care, ${ }^{9}$ Hatton, ${ }^{22}$ and Bhattacharya ${ }^{13,14}$ and is due to the decrease in effective volume available to the surfactants. We also notice that $\mathrm{CMC}$ increases with increasing temperature, a characteristic feature of these models with a LJ type of interaction noted earlier, ${ }^{14,32}$ and CMC very sensitively depends on the chain length. ${ }^{14,33}$

\section{B. Autocorrelation function $A(\tau)$}

Before we present the simulation results for the cluster distributions and shapes of miceller aggregates, it is worthwhile discussing the details of obtaining reliable data from the simulation. We used the autocorrelation function to monitor the kinetics of the aggregates. ${ }^{17}$ The tracer autocorrelation function is defined as

$$
A(\tau)=\frac{\langle N(t+\tau) N(t)\rangle-\langle N(t)\rangle^{2}}{\left\langle N^{2}(t)\right\rangle-\langle N(t)\rangle^{2}}
$$

where for a given $\tau$, the averages $\langle\cdot$.$\rangle are taken over all the$ chains in the system and for all possible time $t$. Here $N(t)$ is the size of the micelle where a tracer chain resides at time $t$. This function has been used by Haliloglu and Mattice, ${ }^{17}$ and by Hatton and co-workers ${ }^{10,22}$ to estimate the length of the time interval $\tau_{c}$ that is needed for the system to evolve from one configuration to another which is statistically independent. It is important to know $\tau_{c}$ to collect data for statistical averaging purposes and to estimate the total length of the simulation time after the system has equilibrated. By definition, $A(0)=1$, and it is expected to decay to zero at a late time. We choose $\tau_{c}$ to be the time when $A(\tau)$ decays to 0.2 . Typically we have run the simulation for $(100-300) \tau_{c}$. Previous $\mathrm{MC}$ studies ${ }^{10,17}$ and stochastic MD study ${ }^{22}$ concentrated on symmetric amphiphiles; where the length of the hydrophobic and hydrophilic segments are the same $\left(h_{x} t_{x}\right.$ in our notation). For concentrations of the amphiphiles which are below the CMC, $A(\tau)$ increases with $X$; but for $X$ beyond $\mathrm{CMC}, A(\tau)$ decreases with increasing concentration. For a larger concentration the average distance between the clusters is less and diffusion of chains from one cluster to the other occurs at a faster rate, which results in a rapid decrease of $A(\tau)$. Our results for asymmetric chains $\left(h_{1} t_{4}\right.$ and $\left.h_{1} t_{6}\right)$ are qualitatively consistent with the above conclusions. Figure 3 shows the variation of $A(\tau)$ as a function of temperature for amphiphiles with $\sigma_{\mathrm{hh}}=2 \sigma_{\mathrm{tt}}$ for total concentration $X=2 \%$. Consistent with previous studies we note that $A(\tau)$ decays faster with $\tau$ at higher temperatures. It is worth pointing out that for the same concentration $X$ and temperature $T$, the decay of $A(\tau)$ is very sensitive to the chain length, especially at a lower temperature. We notice from Fig. 3 that as the chain length is changed from 5 to 7 (inset), $\tau_{c}$ increases from 800 to 1600 . This shows why it becomes increasingly difficult to carry out a simulation for larger chains. Figure 4 shows the variation of $A(\tau)$ as a function of the total concentration $X$. Our choice of concentrations $X=1 \%, 2 \%$, and 


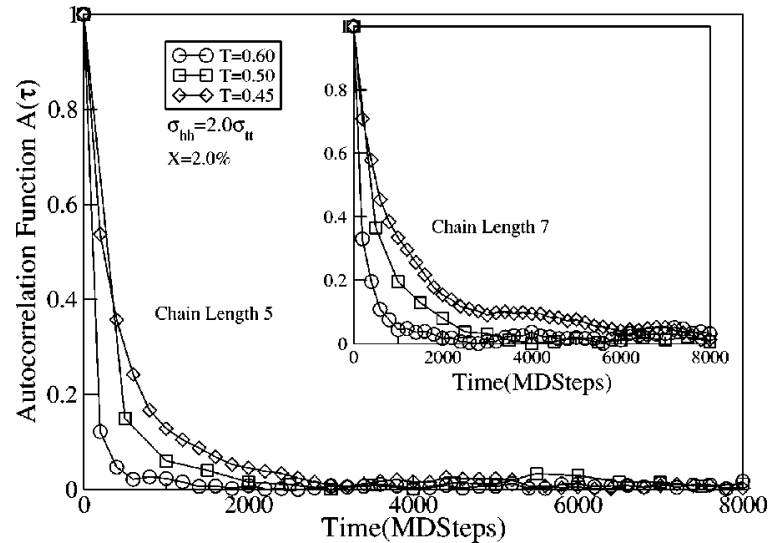

FIG. 3. The behavior of $A(\tau)$ for $h_{1} t_{4}$ and $h_{1} t_{6}$ (inset) at $2 \%$ chain concentration for $\sigma_{\mathrm{hh}}=2 \sigma_{\mathrm{tt}}$ for three different temperatures $T=0.6(\bigcirc), T=0.50$ $(\square)$, and $T=0.45(\diamond)$, respectively.

$3 \%$ are all beyond the $\mathrm{CMC}$ at this particular temperature. We notice that $A(\tau)$ decreases faster for larger concentrations.

Finally, we show the influence of the amphiphilic geometry on $A(\tau)$ in Fig. 5. Notice that the autocorrelation has a faster decay for the amphiphiles with a larger head group area. This behavior could also be inferred from Figs. 1-4. We observe from Figs. 1 and 2 that an amphiphile with a larger head can be looked at as an amphiphile with a smaller head at a higher temperature. That $A(\tau)$ decays faster at a higher temperature (Fig. 3) would also imply a faster decay for large-head amphiphiles, as shown in Fig. 5. Alternately, we can think that the increased head size effectively increases the available volume of the surfactants. Therefore, a faster decay of $A(\tau)$ for the large-head amphiphiles is also consistent with Fig. 4. We have used these autocorrelation functions to determine how often to collect data for statistical averaging purposes. Data is taken at MD time intervals bigger than $\tau_{c}$.

\section{Cluster shapes and distribution}

During the simulation we have monitored the cluster distribution $\left(X_{N} / N\right)$ for several different concentrations and

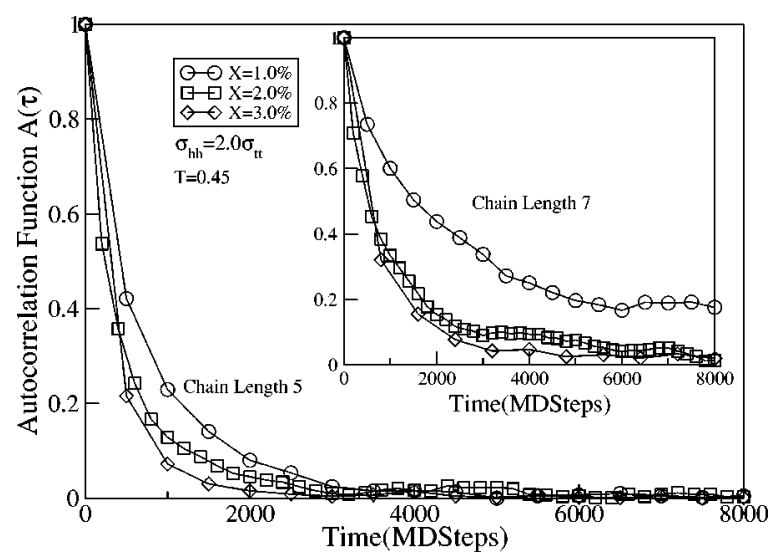

FIG. 4. The behavior of $A(\tau)$ for $h_{1} t_{4}$ and $h_{1} t_{6}$ (inset) at $2 \%$ chain concentration with $\sigma_{\mathrm{hh}}=2$ for three different concentrations $X=1 \%(\bigcirc), X=2 \%$ $(\square)$, and $X=3 \%(\diamond)$, respectively.

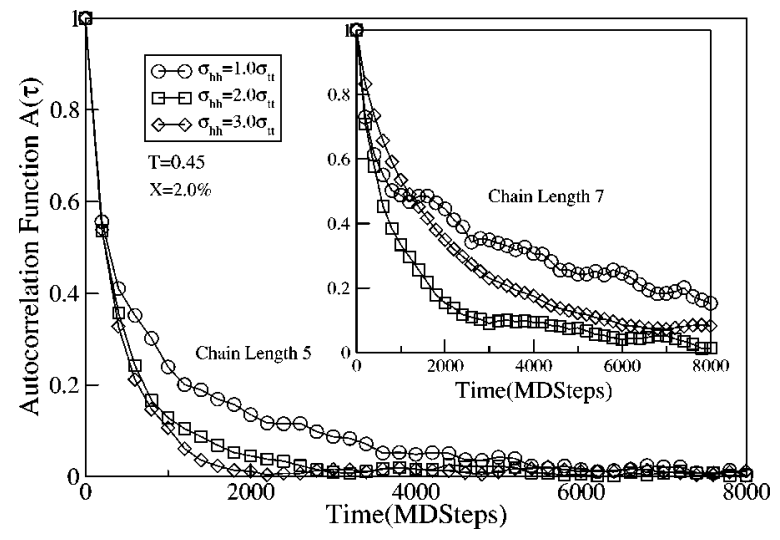

FIG. 5. The behavior of $A(\tau)$ at $T=0.45$ and $X=2 \%$ for three different geometries $\sigma_{\mathrm{hh}}=\sigma_{\mathrm{tt}}(\bigcirc), \sigma_{\mathrm{hh}}=2 \sigma_{\mathrm{tt}}(\square)$, and $\sigma_{\mathrm{hh}}=3 \sigma_{\mathrm{tt}}(\diamond)$ for $h_{1} t_{4}$ and $h_{1} t_{6}$ (inset), respectively.

temperatures for the amphiphiles $h_{1} t_{4}$ and $h_{1} t_{6}$, respectively. We have used the ratio of the two principal moment of inertia to characterize the miceller shapes. Components of the inertia matrix $I_{\alpha \beta}$ are defined as

$$
I_{\alpha \beta}=\sum_{i=1}^{n} m_{i}\left(X_{\mathrm{CM}}^{\alpha}-x_{i}^{\alpha}\right)\left(X_{\mathrm{CM}}^{\beta}-x_{i}^{\beta}\right),
$$

where $X_{\mathrm{CM}}^{j}$ and $x_{i}^{j}$ are the $j$-th co-ordinates of the center of mass of the cluster and the $i$-th particle of the cluster, respectively. In two dimensions there are only three independent components: $I_{x x}, I_{y y}$, and $I_{x y}$. We diagonalize the inertia tensor which has the following two roots (principal moment of inertia):

$$
I_{1,2}=\frac{1}{2}\left(I_{x x}+I_{y y} \pm \sqrt{\left(I_{\mathrm{xx}}-I_{\mathrm{yy}}\right)^{2}+4 I_{x y}^{2}}\right) .
$$

We have used $l_{1,2}=\sqrt{I_{1,2} /\left(I_{1}+I_{2}\right)}$ as the definition of characteristic lengths and used the ratio $\eta=l_{1} / l_{2}$ as a measure of the sphericity of the micelle. For a perfectly spherical (circular in two dimensions) micelles $\eta=1.0$. In reality, since it is almost impossible to have a perfectly spherical micelle, the parameter $\eta>1$. Figure 6 shows the effect of the different head group geometry on the cluster distribution $X_{N} / N$ (bottom) and the shape parameter $\eta$ (top) at $X=0.2, T=0.45$ for $h_{1} t_{6}$. We notice that for $\sigma_{\mathrm{hh}}=\sigma_{\mathrm{tt}}$ the cluster distribution is relatively flat with a larger probability for the occurrence of larger clusters. As we increase $\sigma_{\text {hh }}$ to $2 \sigma_{\text {hh }}$ and $3 \sigma_{\text {hh }}$ the cluster distribution becomes progressively sharper, the occurrence of larger clusters becomes rarer, and the peak of the distribution shifts toward a smaller value.

We would like to relate our results with the dimensionless packing parameter in two dimensions. In three dimensions (3D), for a spherical micelle of radius $R$ containing $n$ chains, self-consistency requires that

$$
n=\frac{4 \pi R^{2}}{a_{0}}=\frac{4 \pi R^{3}}{3 v}
$$

where $v$ is the average volume occupied by a single surfactant and $a_{0}$ is the average area occupied by the head monomer in the micelle. This yields $R=3 v / a_{0}$, so that only for 


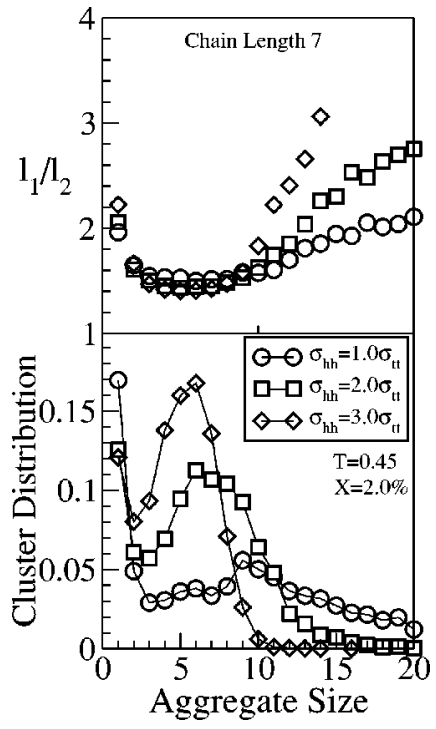

FIG. 6. Variation of the shape parameter $\eta$ (top) and the cluster distribution $X_{N} / N$ (bottom) as a function of the cluster size $N$ for three different head group geometries $\sigma_{\mathrm{hh}}=\sigma_{\mathrm{tt}}(\bigcirc), \sigma_{\mathrm{hh}}=2 \sigma_{\mathrm{tt}}(\square)$, and $\sigma_{\mathrm{hh}}=3 \sigma_{\mathrm{tt}}(\diamond)$, respectively.

$\lambda_{3 \mathrm{D}}=v / a_{0} l_{c}<\frac{1}{3}$ spherical micelles are formed. ${ }^{1}$ When translated in two dimensions, it is straightforward to see that this self-consistency condition becomes

$$
n=\frac{2 \pi R}{l_{0}}=\frac{\pi R^{2}}{s},
$$

where $s$ is the average area occupied by a single surfactant and $l_{0}$ is the average length occupied by the head monomer in a micelle in two dimensions. From this we get $\lambda_{2 \mathrm{D}}$ $=s / l_{0} l_{c}<\frac{1}{2}$, the packing condition for circular micelles. We can express the dimensionless packing parameter in two dimensions in terms of the number of tail monomers $n_{t}, \sigma_{\mathrm{hh}}$ and $\sigma_{\mathrm{tt}}$ in the following manner. We can approximate the area occupied by the tails of the amphiphilic molecule as $s$ $\sim n_{t}^{\alpha}\left(\sigma_{\mathrm{tt}}\right)^{2}$, where $\alpha \geqslant 1 \quad(\alpha=1$ for compact 1D or 2D geometries and in this case would be a fractal dimension greater than 1 to account for the void inside the micelle). The effective chain length inside the micelle $l_{c}=n_{t}^{0.5} \sigma_{\mathrm{tt}}$, assuming that the end to end distance for the tail monomers are described by a random walk. We expect the head group area (arc) $l_{0}$ to be proportional to $\sigma_{\mathrm{hh}}$. Thus, $\lambda_{2 \mathrm{D}}$ $\sim n_{t}{ }^{\alpha-0.5}\left(\sigma_{\mathrm{tt}} / \sigma_{\mathrm{hh}}\right)$. Therefore, $\lambda_{2 \mathrm{D}}$ can be altered by either varying the length of the tail $n_{t}$, or the ratio $\sigma_{\mathrm{tt}} / \sigma_{\mathrm{hh}}$.

We now look at the corresponding shape parameter $\eta$. In general, as a function of the cluster size, $\eta$ has a form of a well, exhibiting a minimum for a certain value of $n$. For smallest $\sigma_{\mathrm{hh}}$, around this minimum, $\eta$ is a slowly varying function of $n$; As we increase the head size, $\eta$ rises rapidly beyond its minimum. A careful look at the plot shows that the value of $\eta$ at the minimum depends, albeit weakly on $\sigma_{\text {hh }}$. Combining the information from the cluster distribution and the shape parameter, we conclude that for a fixed length of the hydrophobic tail $n_{t}$, an increase of the optimal head group area would reduce the value of $\lambda_{2 \mathrm{D}}$ to produce sharper cluster distributions with micelles which are more spherical. Similar results can be obtained by keeping the hydrophilic

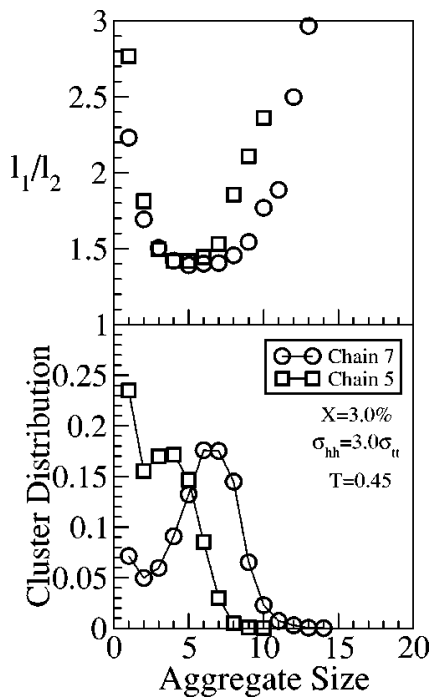

FIG. 7. Variation of the shape parameter $\eta$ (top) and the cluster distribution $X_{N} / N$ (bottom) as a function of the cluster size $N$ for two different chain lengths $m=5$ and 7 , respectively.

head the same and varying the hydrophobic unit $n_{t}$. Figure 7 shows the same variation for two different chain lengths $m$ $=5$ and 7. For both the chains we have chosen $\sigma_{\mathrm{hh}}=3 \sigma_{\mathrm{tt}}$; the hydrophobic tail units are varied to be 4 and 6 , respectively. Evidently $\lambda_{2 D}$ for the smaller chain is smaller and therefore it has a sharper variation compared to the longer chain. Likewise, the peak in the cluster distribution shifts at a lower value for the shorter chain. To make these arguments more pictorial, we have shown two typical snapshots for a small and a large head group in Fig. 8. A change of $\sigma_{\mathrm{hh}}$

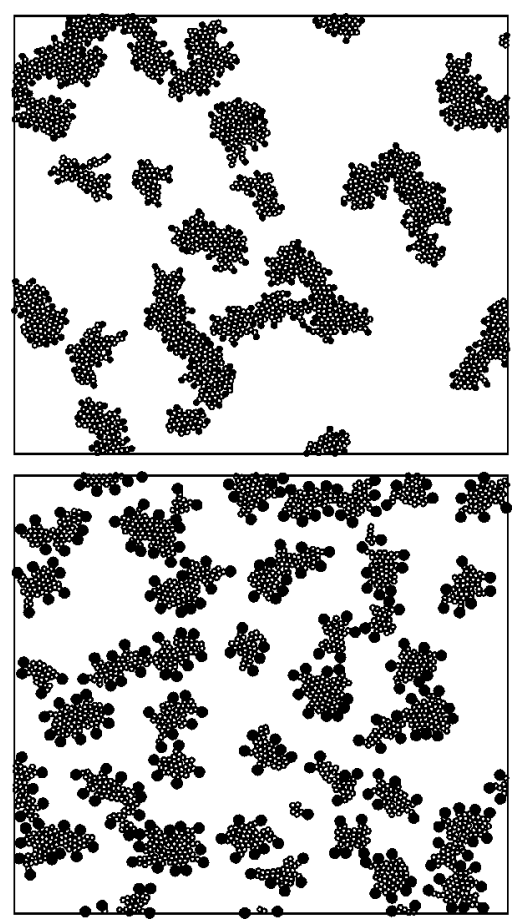

FIG. 8. Snapshot of the amphiphilic aggregates at $T=0.45, X=2 \%$ for $h_{1} t_{6}$ using $\sigma_{\mathrm{hh}}=\sigma_{\mathrm{tt}}$ (top) and $\sigma_{\mathrm{hh}}=2 \sigma_{\mathrm{tt}}$ (bottom). The latter tends to form more spherical and smaller micelles with a sharper cluster distribution. 


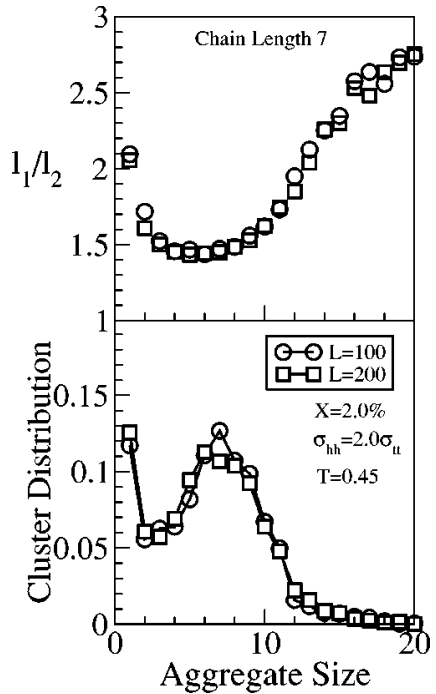

FIG. 9. Variation of shape parameter $\eta$ (top) and the cluster size distribution (bottom) from simulation of $h_{1} t_{6}$ at $X=2 \%$ and $T=0.45$ using two different system sizes. The symbols $\bigcirc$ and $\square$ correspond to the choices of the simulation box $L=100 \sigma_{\mathrm{tt}}$ and $200 \sigma_{\mathrm{tt}}$ containing 200 and 800 amphiphiles, respectively.

$=\sigma_{\mathrm{tt}}$ (top) to $\sigma_{\mathrm{hh}}=2 \sigma_{\mathrm{tt}}$ (bottom) produces a huge variation in shapes and sizes of the micelles.

\section{The effect of the simulation box}

Finally, we investigated the finite size effect of the simulation box on the cluster distribution and shapes. The largest cluster size is limited by the total number of chains and therefore it is possible that the occurrence of large clusters will be affected by the box size. We have compared the results obtained from simulation boxes $L=100 \sigma_{\mathrm{tt}}$ and $L$ $=200 \sigma_{\mathrm{tt}}$; respectively, for identical parameters. A comparison is shown for the cluster distribution and the shape parameter for the set $X=2 \%$ and $T=0.45$ in Fig. 9. We notice that cluster distribution and shape up to cluster size 20 remain practically unaffected by the size of the simulation box which establishes that the observed dependence of shape and sizes on $\sigma_{\mathrm{hh}}$ is established beyond any doubt. We have checked this conclusion to be true for other choices of temperature and concentrations. It is worth mentioning at this point that we have chosen a NVT ensemble which does not allow us to obtain the free volume released out due to micelle formation. It will be interesting to study finite size effects in a NPT ensemble which will accommodate any free volume change due to packing constraints.

\section{CONCLUSION}

In summary, we have studied the role of the head group geometry in amphiphilic self-assembly for a bead-spring model of flexible amphiphiles using Brownian dynamics simulation. Usually in simulation studies based on a lattice and off-lattice models different characteristics of amphiphilic self-assembly is investigated as a function of concentration and temperature and chain length. In this paper we focused on a systematic investigation of geometric effects in amphiphilic self-assembly. That this is very important has been studied analytically under certain approximations by Israelachvili and co-workers. Here we demonstrate for a semirealistic model how the shapes and sizes can be controlled by the proper tuning of the head-to-tail ratio, or by changing the size/length of the hydrophilic and hydrophobic segments. We find that the geometric effects are rather nontrivial and a simulation based knowledge can be very useful for nanomasking and other surfactant mediated templating methods. In order to make more direct contact with experiments a three dimensional simulation is in progress and will be reported in a separate publication. ${ }^{34}$ We are also using this model to study grafting properties of surfactants in ferro colloids. ${ }^{35}$

\section{ACKNOWLEDGMENT}

The research reported here is supported in part by a grant from the National Science Foundation NIRT (ENG/ECS and CISE/EIA) under Grant No. 0103587.

${ }^{1} \mathrm{~J}$. Israelachvili, Intermolecular and Surface Forces, 2nd ed. (Academic, London, 1992).

${ }^{2}$ S. A. Safran, Statistical Thermodynamics of Surfaces, Interfaces, and Membranes (Addison-Wesley, New York, 1994).

${ }^{3}$ M. Haupt, S. Miller, A. Ladenburger, R. Sauer, K. Thonnke, J. P. Spatz, S. Reithmuller, M. Moller, and F. Barnhard, J. Appl. Phys. 91, 6057 (2002).

${ }^{4}$ B. Alberts et al., Molecular Biology of the Cell, 4th ed. (Gerland Science, New York, 2002).

${ }^{5}$ J. J. Kasianowicz, E. Brandin, D. Branton, and D. Deamer, Proc. Natl. Acad. Sci. U.S.A. 93, 13770 (1996).

${ }^{6}$ C. Sangregorio, J. K. Wiemann, C. J. O’Connor, and Z. Rosenzweig, J. Appl. Phys. 85, 5699 (1999).

${ }^{7}$ J. Israelachvili, D. J. Mitchell, and B. W. Ninham, J. Chem. Soc., Faraday Trans. 2 72, 1525 (1976).

${ }^{8}$ R. G. Larson, L. E. Scriven, and H. T. Davis, J. Chem. Phys. 83, 2411 (1985); R. G. Larson, ibid. 89, 1642 (1988); 91, 2479 (1989); 96, 7904 (1992); J. Phys. II 6, 1441 (1996).

${ }^{9}$ C. M. Care, J. Phys. C 20, 689 (1987); J. Chem. Soc., Faraday Trans. 83, 2905 (1987); D. Brindle and C. M. Care, ibid. 88, 2163 (1994); J.-C. Desplat and C. M. Care, Mol. Phys. 87, 441 (1996).

${ }^{10}$ P. H. Nelson, G. C. Rutledge, and T. A. Hatton, J. Chem. Phys. 107, 10777 (1997).

${ }^{11}$ A. T. Bernardes, V. B. Henriques, and P. M. Bisch, J. Chem. Phys. 101, 645 (1994); J. Phys. II 6, 169 (1996)

${ }^{12}$ A. D. Mackie, A. Z. Panagiotopolous, and I. Szliefer, Langmuir 13, 5022 (1997).

${ }^{13}$ A. Bhattacharya and S. D. Mahanti, J. Phys.: Condens. Matter 12, 6141 (2000).

${ }^{14}$ A. Bhattacharya and S. D. Mahanti, J. Phys.: Condens. Matter 13, L861 (2001).

${ }^{15}$ C. S. Shida and V. B. Henriques, J. Chem. Phys. 115, 8655 (2001).

${ }^{16}$ A. Milchev, A. Bhattacharya, and K. Binder, Macromolecules 6, 1881 (2001).

${ }^{17}$ T. Haliloglu and W. L. Mattice, Chem. Eng. Sci. 49, 2851 (1993).

${ }^{18}$ D. R. Rector, F. van Swol, and J. R. Henderson, Mol. Phys. 82, 1009 (1994).

${ }^{19}$ B. Palmer and J. Liu, Langmuir 12, 746 (1996); 12, 6015 (1996).

${ }^{20}$ P. K. Maiti, Y. Lansac, M. A. Glaser, N. A. Clark, and Y. Rouaunt, Langmuir 18, 1908 (2002).

${ }^{21}$ J.-B. Maillet, V. Lachet, and P. V. Coveney, Phys. Chem. Chem. Phys. 1, 5277 (1999).

${ }^{22}$ F. K. von Gottberg, K. A. Smith, and T. A. Hatton, J. Chem. Phys. 106, 9850 (1997).

${ }^{23}$ S. Bogusz, R. M. Venable, and R. Pastor, J. Phys. Chem. 105, 8312 (2001).

${ }^{24}$ A. Bhattacharya, S. D. Mahanti, and A. Chakrabarti, Phys. Rev. Lett. 80, 333 (1998).

${ }^{25}$ A. Bhattacharya and A. Milchev, Phys. Rev. E 66, 041806 (2002). 
${ }^{26}$ G. S. Grest and K. Kremer, Phys. Rev. A 33, 3628 (1986).

${ }^{27}$ W. F. van Gunsteren and H. J. C. Berendsen, Mol. Phys. 45, 637 (1982).

${ }^{28}$ E. Ruckenstein and R. Nagarajan, J. Phys. Chem. 79, 2622 (1975).

${ }^{29}$ R. Nagarajan and E. Ruckenstein, Langmuir 7, 2934 (1980).

${ }^{30}$ A. Ben-Naim and F. H. Stillinger, J. Phys. Chem. 84, 2872 (1980).

${ }^{31}$ F. H. Stillinger and A. Ben-Naim, J. Chem. Phys. 74, 2510 (1981).
${ }^{32}$ A. Floriano, E. Caponetti, and A. Z. Panagiotopolous, Langmuir 15, 3143 (1998).

${ }^{33}$ Surfactants and Polymers in Aqueous Solution, edited by B. Jönsson, B. Lindman, K. Holmberg, and B. Kronberg (Wiley, New York, 1998), see Chaps. 2 and 4.

${ }^{34} \mathrm{G}$. Bourov and A. Bhattacharya (unpublished).

${ }^{35}$ K. Chen, A. F. Bakuzis, and W. Luo (unpublished). 